\title{
Reciprocal zebrafish-medaka hybrids reveal maternal control of zygotic genome activation timing
}

Krista R. Gert ${ }^{1,2}$, Luis Enrique Cabrera Quio, ${ }^{1,2}$, Maria Novatchkova ${ }^{1}$, Yixuan Guo ${ }^{3}$, Bradley R. Cairns $^{3}$, and Andrea Pauli ${ }^{1 *}$

${ }^{1}$ Research Institute of Molecular Pathology (IMP), Vienna BioCenter (VBC), Campus-ViennaBiocenter 1, 1030 Vienna, Austria

${ }^{2}$ Vienna BioCenter PhD Program, a Doctoral School of the University at Vienna and the Medical University of Vienna, 1030 Vienna, Austria

${ }^{3}$ Howard Hughes Medical Institute, Department of Oncological Sciences and Huntsman Cancer Institute, University of Utah School of Medicine, Salt Lake City, Utah 84112, USA

*Corresponding author: andrea.pauli@imp.ac.at

Keywords: hybrids, fertilization, zygotic genome activation, medaka, zebrafish

\begin{abstract}
After fertilization, the sperm and egg contribute unequally to the newly formed zygote. While the sperm contributes mainly paternal DNA, the egg provides both maternal DNA and the bulk of the future embryonic cytoplasm. Most embryonic processes (like the onset of zygotic transcription) depend on maternally-provided cytoplasmic components, and it is largely unclear whether paternal components besides the centrosome play a role in the regulation of early embryogenesis. Here we report a reciprocal zebrafish-medaka hybrid system as a powerful tool to investigate paternal vs. maternal influence during early development. By combining expression of zebrafish Bouncer on the medaka egg with artificial egg activation, we demonstrate the in vitro generation of paternal zebrafish $\mathrm{x}$ maternal medaka (reripes) hybrids. These hybrids complement the previously established paternal medaka $x$ maternal zebrafish (latio) hybrids (Herberg et al., 2018). As proof of concept, we investigated maternal vs. paternal control of zygotic genome activation (ZGA) timing using this reciprocal hybrid system. RNA-seq analysis of the purebred fish species and hybrids revealed that the onset of ZGA is primarily governed by the egg. Overall, our study establishes the reciprocal zebrafishmedaka hybrid system as a versatile tool to dissect parental control mechanisms during early development.
\end{abstract}

\section{Introduction}

Our limited ability to distinguish maternal and paternal components during development has hindered investigation into many fundamental mechanisms that govern embryogenesis. Researchers have long strived for an ideal system with which to accomplish this on the gene, transcript, and protein levels. A traditional approach to accomplish this beyond studies at the single-gene level has been to cross parents carrying different single nucleotide polymorphisms (SNPs) (Harvey et al., 2013; Petkov et al 2004; Lilue et al., 2018. However, this methodology is restricted to the subset of loci containing SNPs. Here, we report a versatile system using zebrafish (Danio rerio) and medaka (Oryzias latipes) hybrids that enables the distinction between maternal vs. paternal origin at the near-genome-wide level.

Zebrafish and medaka are both well-established genetic models that can be housed in similar environmental conditions and undergo relatively fast early development. They are externally fertilizing and produce gametes and embryos that are amenable for live-cell imaging (FurutaniSeiki \& Wittbrodt, 2004). Importantly, zebrafish and medaka do not interbreed, and medaka sperm cannot fertilize zebrafish eggs in vitro (Herberg et al., 2018). As zebrafish and medaka are two evolutionarily distant species ( 110 MYR) (Wittbrodt et al., 2002), their genomes differ in sequence, size, and chromosome number. The medaka genome is $0.7 \mathrm{~Gb}$, around half the size of that of zebrafish $(1.4 \mathrm{~Gb})$ and comprises 24 chromosomes including sex chromosomes, 
whereas the zebrafish genome constitutes 25 autosomes. In spite of these differences, both genomes contain a similar number of genes ( 25,000) (Kasahara et al 2007; Howe et al 2013). Because even conserved genes differ in nucleotide sequence, the vast majority of sequencing-derived reads from zebrafish-medaka hybrids should be unequivocally mappable to either genome. This makes the artificial hybridization between zebrafish and medaka a unique tool with which to address open questions of paternal and maternal control, conserved developmental mechanisms, as well as hybrid incompatibilities.

We previously reported that expressing the medaka homolog of Bouncer, an egg-expressed factor essential for fertilization, on zebrafish eggs was sufficient to allow fertilization of these eggs by medaka sperm (Herberg et al, 2018). Following traditional hybrid nomenclature in which the paternal name is followed by the maternal one, we fused the species names of Danio rerio and Oryzias latipes to name the resulting hybrid embryos "latio" (paternal genome from $O$. latipes; maternal genome from $D$. rerio). While latio hybrids alone are already a useful tool, the reverse hybrid, "reripes" (paternal genome from $D$. rerio; maternal genome from $O$. latipes), has never been generated, but would serve as the ideal comparison to latio hybrids and reveal the effects of having each species as each parent. Here we establish an efficient protocol to produce reripes hybrids. Furthermore, we use our newly established reciprocal hybrid system to address the long-standing question of whether the timing of zygotic genome activation is under cytoplasmic (maternal) vs. genomic control.

\section{Results}

We investigated whether reripes hybrid embryos could be produced in in vitro fertilization experiments with zebrafish sperm and medaka eggs overexpressing the zebrafish Bouncer homolog, using a strategy similar to the one employed for generating latio embryos (Herberg et al., 2018). While both fish are teleosts, medaka and zebrafish differ in their egg-laying behavior and produce telolecithal eggs that have important physiological differences. Firstly, while unfertilized zebrafish eggs can be readily obtained from females in vitro (Westerfield, 2007), efficient collection of unfertilized medaka eggs requires mating of medaka females with infertile Oryzias curvinotus x Oryzias latipes hybrid males (Kamei et al., 2007). Secondly, in both organisms, the sperm contacts the egg only through a small opening in the protective envelope (chorion) around the egg. This opening, the micropyle, is a narrow canal through which the sperm must swim to reach the egg membrane. Because medaka and zebrafish sperm are similar in size (Wolenski \& Hart, 1987; Iwamatsu et al., 1993; Iwamatsu et al., 1997), the medaka micropyle should be able to accommodate zebrafish sperm. However, whether zebrafish sperm can indeed locate and pass through the medaka micropyle has not been tested. Thirdly, zebrafish sperm and eggs are activated upon contact with water, a hypotonic solution that induces sperm motility and renders the egg competent for fertilization (Cosson, 2004; Westerfield, 2007). In contrast, medaka eggs activate upon contact with sperm, while the sperm also acquires motility through contact with water (Iwamatsu et al., 1991).

We thus first investigated whether zebrafish sperm can both locate and pass through the medaka micropyle and thereby contact the egg membrane. Live-cell imaging of in vitro fertilization using MitoTracker-labeled zebrafish sperm and wild-type (medaka Bouncerexpressing) as well as transgenic zebrafish Bouncer-expressing medaka eggs revealed that zebrafish sperm are indeed able to find and enter the medaka micropyle. However, no sperm were observed to enter the egg after reaching the micropylar pit, even after several minutes and irrespective of the origin of the expressed Bouncer protein (Fig. 1A). In concordance with these observations, in vitro fertilization (IVF) experiments with zebrafish sperm incubated with medaka eggs did not produce any fertilized eggs (Fig. 1B). In contrast, medaka sperm labeled with MitoTracker were able to enter the medaka egg after $\sim 2$ minutes (Fig. 1A) and efficiently fertilized eggs in IVF (Fig. 1B), as expected for conspecific sperm. Thus, while expression of medaka Bouncer on the zebrafish egg enables medaka sperm to enter, resulting in the 
bioRxiv preprint doi: https://doi.org/10.1101/2021.11.03.467109; this version posted November 4, 2021. The copyright holder for this

preprint (which was not certified by peer review) is the author/funder, who has granted bioRxiv a license to display the preprint in perpetuity. It is made available under aCC-BY-NC 4.0 International license.

generation of latio hybrid embryos (Herberg et al., 2018), expression of zebrafish Bouncer on medaka eggs is not sufficient for zebrafish sperm to enter medaka eggs.
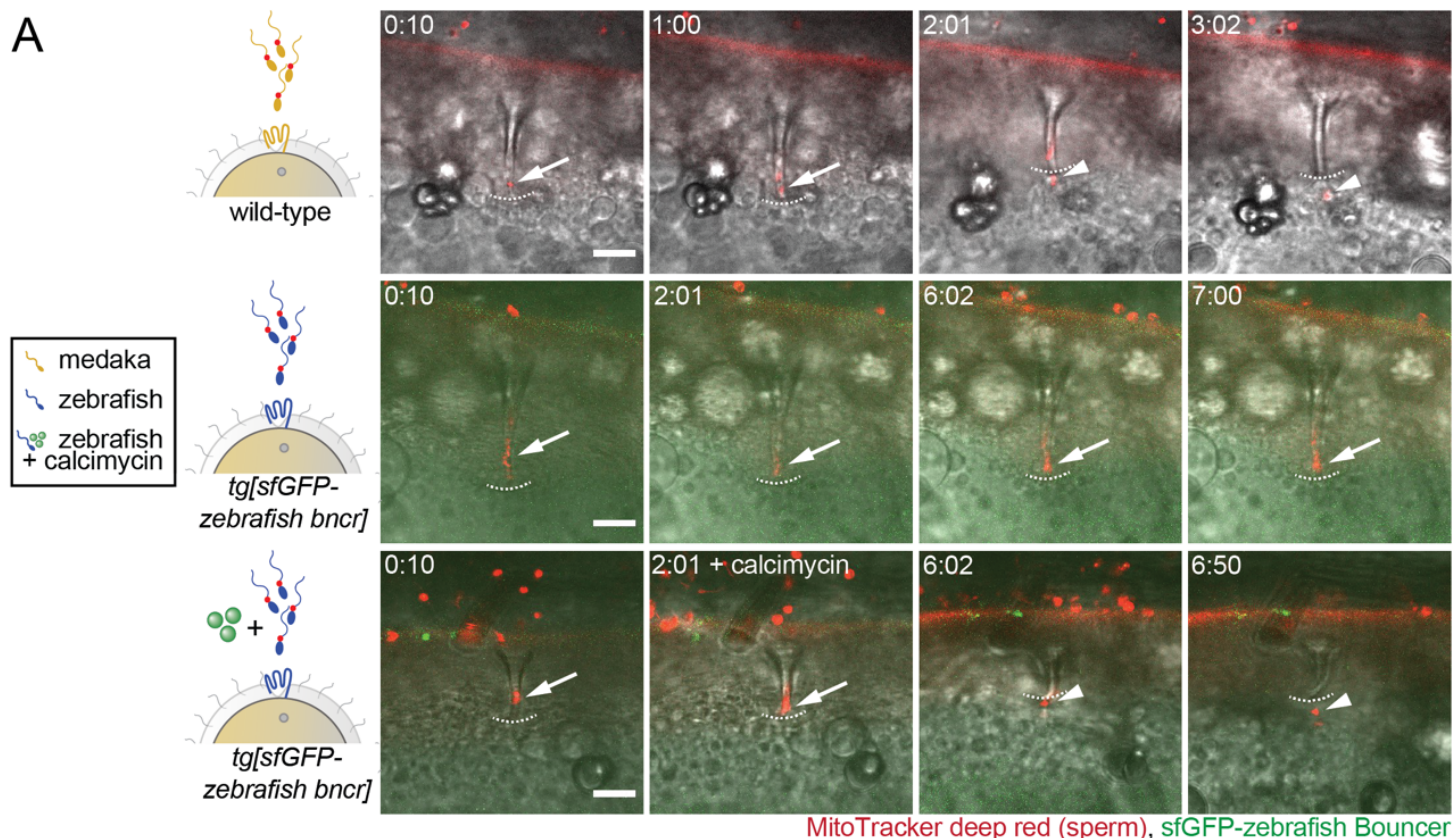

MitoTracker deep red (sperm), sfGFP-zebrafish Bouncer

B

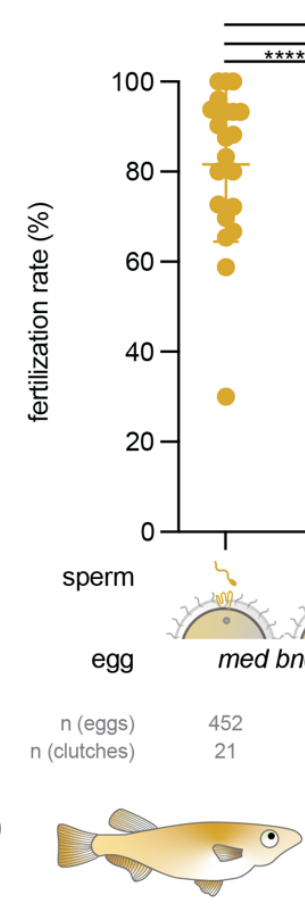

+ Oryzias latipes
X

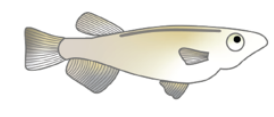

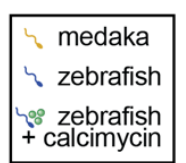

C

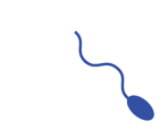

zebrafish sperm only
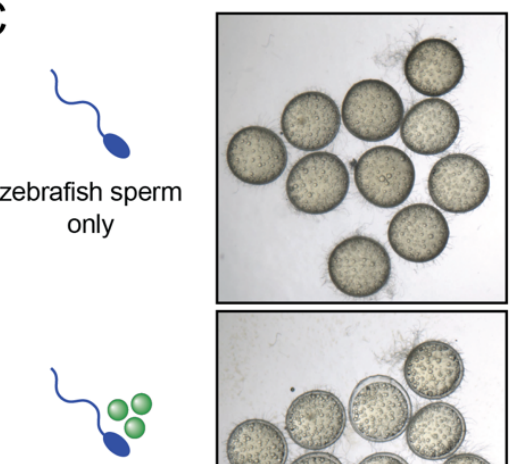

zebrafish sperm + calcimycin

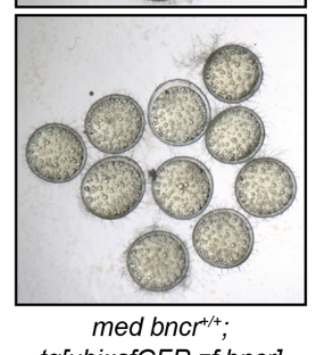

$\operatorname{tg}[u b i:: s f G F P-z f$ bncr]
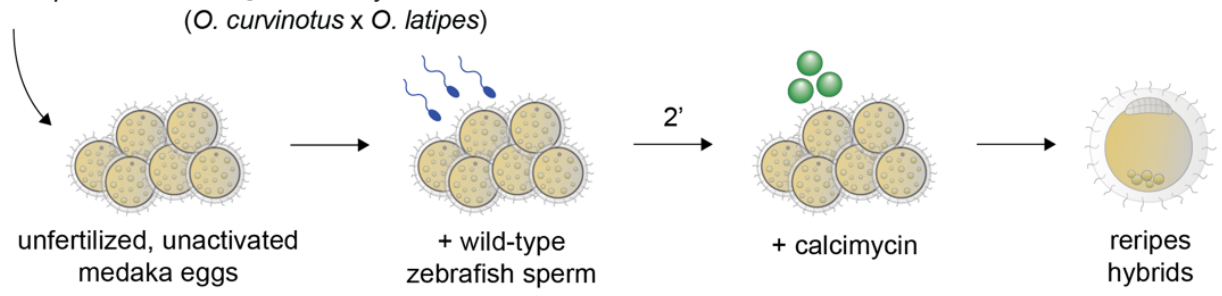


\section{Figure 1. Zebrafish sperm can fertilize zebrafish Bouncer-expressing medaka eggs} upon artificial activation by calcimycin.

(A) Time-lapse images (20X) of MitoTracker-labeled sperm (red) incubated with medaka eggs. (Top) Medaka sperm efficiently fertilize wild-type medaka eggs and can be observed inside the egg $\sim 2$ minutes post-sperm addition. (Middle) Zebrafish sperm find and enter the micropyle of medaka eggs expressing zebrafish Bouncer (green), but do not fuse with the egg. (Bottom) Addition of calcimycin $\sim 2$ minutes post-sperm addition induces egg activation and allows zebrafish sperm to fuse with zebrafish Bouncer-expressing medaka eggs. White arrows indicate sperm inside the micropyle. Dashed white lines demarcate the base of the micropyle. White arrowheads indicate sperm inside the egg after it has passed the base of the micropyle and fused with the egg membrane.

(B) In vitro fertilization with medaka sperm and wild-type medaka eggs results in high fertilization rates (>60\%). Zebrafish sperm alone are unable to fertilize zebrafish Bouncerexpressing medaka eggs. Addition of calcimycin after zebrafish sperm addition enables fertilization of both wild-type and zebrafish Bouncer-expressing eggs. (Kruskal-Wallis test with Dunn's multiple comparisons test: ${ }^{* * *}$ adj. $p<0.0001,{ }^{* * *} \operatorname{adj} . \mathrm{p}=0.0004,{ }^{* *} \operatorname{adj} . \mathrm{p}=$ 0.005).

(C) Medaka eggs, even when expressing zebrafish Bouncer, remain unactivated (top) in the presence of zebrafish sperm, as seen by the darker-colored cytoplasm and chorion tightly apposed to the egg membrane. Calcimycin addition induces artificial activation of medaka eggs (bottom), evident by the clearer cytoplasm and gradual lifting of the chorion.

(D) Workflow schematic depicting generation of reripes hybrids.

Interestingly, we observed that medaka eggs exposed to zebrafish sperm not only remained unfertilized, but also did not undergo activation (Fig. 1C). These observations led us to hypothesize that zebrafish sperm may be unable to activate medaka eggs and therefore are unable to fertilize them even in the presence of zebrafish Bouncer. Medaka eggs in calciumcontaining media can be activated artificially using the calcium ionophore A23187 (calcimycin) (Ridgway, Gilkey, \& Jaffe, 1977; Gilkey et al., 1978), a divalent cation ionophore that allows passage of calcium across membranes (Luckasen, White, \& Kersey, 1974). Remarkably, when zebrafish Bouncer-expressing medaka eggs were incubated with zebrafish sperm followed by the addition of calcimycin, the eggs were activated and able to be fertilized by zebrafish sperm (Fig. 1A-C). While this does not strictly depend on expression of zebrafish Bouncer, fertilization rates were significantly higher in the presence of zebrafish vs. medaka Bouncer (Fig. 1B). We therefore conclude that combining expression of zebrafish Bouncer on medaka eggs with calcimycin treatment enables the efficient generation of reripes hybrids (Fig. 1D).

The successful generation of reripes hybrids allowed us to compare development and gene expression in reripes and latio hybrids, which have identical genome content (haploid for both zebrafish and medaka genomes) yet differ in the origin of their maternal and paternal genomes as well as maternally-provided cytoplasm (Fig. 2A). Overall development of reripes hybrids follows that of purebred medaka embryos (Fig. 2B). However, most reripes embryos arrest and begin to decompose before 16 hours post-fertilization (hpf), coinciding with the time of gastrulation (Iwamatsu, 2004). Reripes hybrids therefore survive for a shorter period of time than latio hybrids, which develop similarly to purebred zebrafish embryos until they arrest at approximately $24 \mathrm{hpf}$ (Herberg et al., 2018) (Fig. 2B). 
A

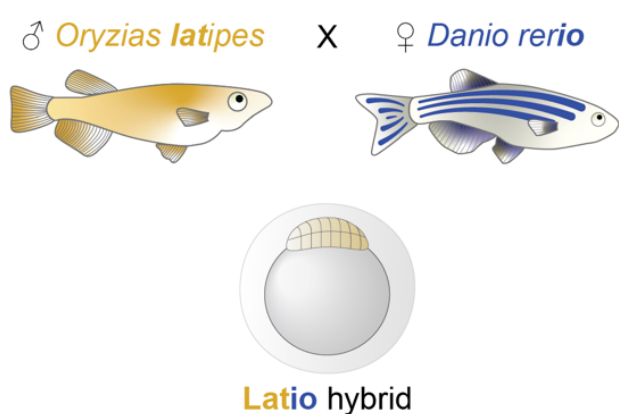

B

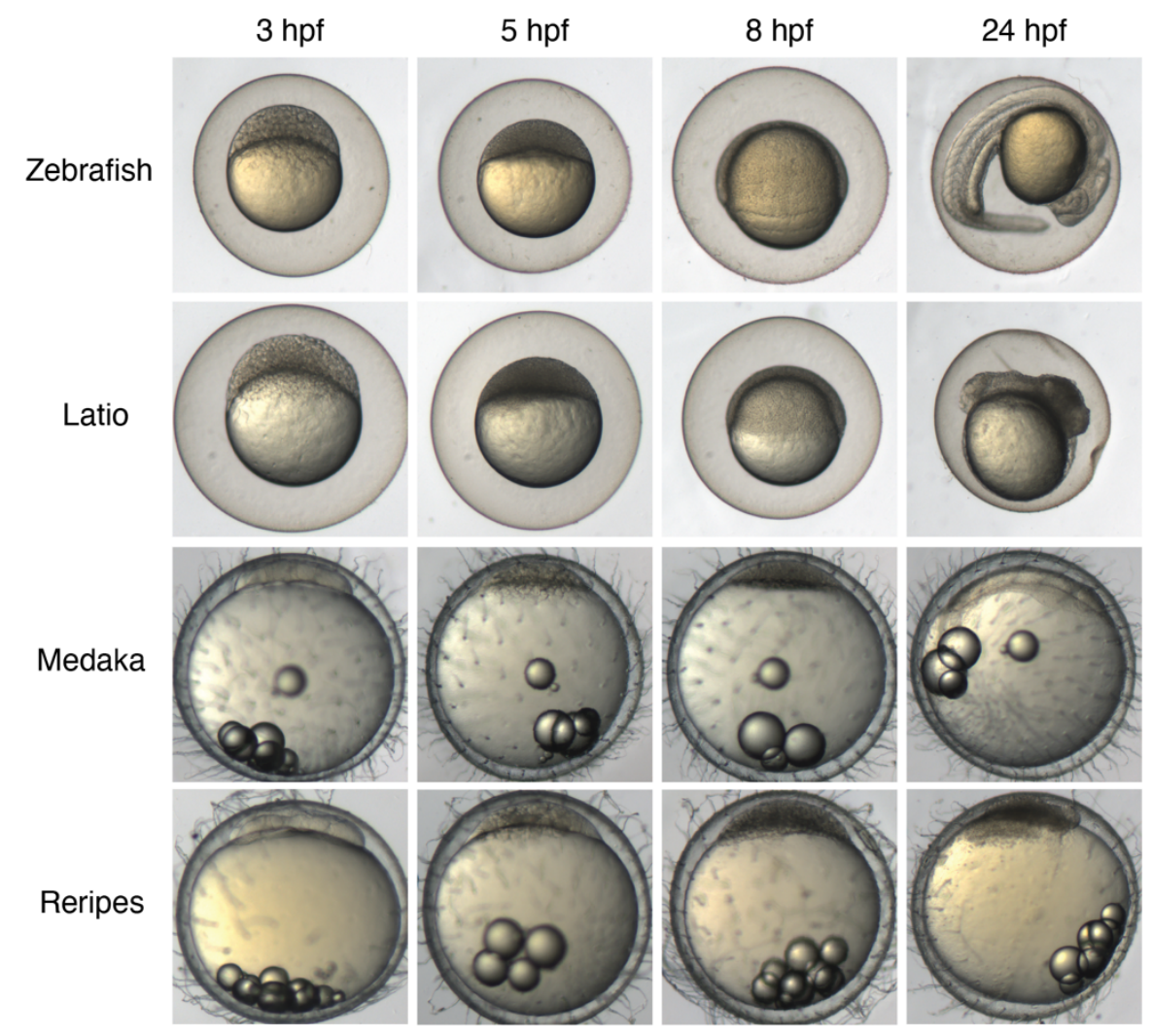

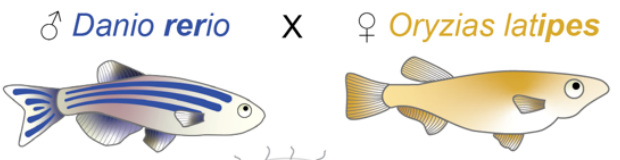

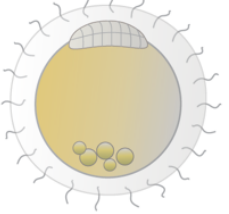

Reripes hybrid

Figure 2. Development of latio and reripes hybrids resembles that of the maternal fish species.

(A) Schematic depicting the naming convention for latio and reripes hybrids, whose names are derived from fusing the paternal and maternal species epithets, respectively.

(B) Bright-field image time course of zebrafish, latio, medaka, and reripes embryos at the corresponding time points for RNA-seq analysis (4X magnification). Overall, latio hybrids resemble zebrafish embryos, while reripes hybrids resemble medaka embryos. However, in both cases, a delay evident by morphology of the hybrid embryo is apparent by 8 hpf. By 24 hpf, both hybrids have arrested development and are necrotic.

Despite hybrid inviability in both cases, latio and reripes hybrids develop past the time of ZGA in both medaka and zebrafish. The major wave of ZGA occurs at $\sim 3 \mathrm{hpf}$ in zebrafish (Vastenhouw et al., 2019), while medaka ZGA takes place later at $\sim 6-8 \mathrm{hpf}$ (Aizawa et al., 2003; Nakamura, et al., 2021). This intrinsic difference between medaka and zebrafish 
development presented us with a unique opportunity to revisit maternal vs. paternal control of ZGA timing. In the medaka-zebrafish hybrid system, the genomic content in both hybrids is identical, but exists in either an early-activating cytoplasm (zebrafish) or a late-activating cytoplasm (medaka). To determine whether the foreign paternal genome is expressed in both hybrids and to compare gene expression dynamics in purebred vs. hybrid embryos, we performed ribosomal RNA-depleted RNA-sequencing on zebrafish, medaka, latio, and reripes samples collected at 3, 5, 8, and $24 \mathrm{hpf}$ (no 24-hpf sample was collected for reripes, given their arrest at $\sim 16 \mathrm{hpf}$ ). Consistent with maternal contribution of mRNAs, reads from the earliest time point mapped to the corresponding maternal genome in both hybrids (Fig. 3A). However, reads from later time points mapped uniquely to regions in zebrafish and medaka genomes for both hybrid samples (Fig. 3A), demonstrating that the zebrafish genome is expressed in the medaka egg (reripes hybrid) and the medaka genome is expressed in the zebrafish egg (latio hybrid). Although only a small fraction of the total number of reads was derived from the paternal genome during the first hours after the onset of ZGA in both hybrids, thousands of paternal genes became expressed at the latest time point analyzed for each hybrid, as evidenced by the $\sim 15-20,000$ genes expressed in purebreds across all time points vs. $\sim 30,000$ genes expressed in late hybrid samples (Fig. 3B). We therefore conclude that genome activation in zebrafish-medaka hybrids occurs genome-wide and that both zebrafish and medaka cytoplasmic components can drive successful activation of the other species' genes.

Because both genomes are actively expressed in both hybrids, we analyzed the timing of ZGA in hybrid embryos. We limited our analysis to zygotically expressed genes, which we defined based on embryonic gene expression time courses of zebrafish (Bhat et al., in preparation) and medaka (Li et al., 2020) (see Materials and Methods). Comparison of the onset of ZGA in hybrid embryos vs. parental species revealed that maternally- and paternally-derived genes became activated at similar times in a given hybrid embryo and followed the maternal timing (Fig. 3C, D). As such, robust onset of paternally-derived zebrafish gene expression was detected in the reripes hybrid at $8 \mathrm{hpf}$, coinciding with the onset of expression of zygotically expressed medaka genes in reripes hybrids, yet delayed compared to zebrafish's regular timing of ZGA at $\sim 3 \mathrm{hpf}$ (Fig. 3C). Along the same lines, onset of paternally-derived medaka gene expression was already detected at $5 \mathrm{hpf}$ in the latio hybrid, which is premature in comparison to ZGA timing in medaka (Fig. 3D).

In order to compare the expression dynamics of both zebrafish and medaka alleles for a given gene, we extended our analysis to the subset of orthologous gene pairs between zebrafish and medaka. In addition to the findings described above, this analysis revealed that in hybrid embryos, both zebrafish and medaka alleles of orthologous genes were in general activated simultaneously (Fig. 4A). (Note that a subset of orthologous genes that are zygotically expressed in zebrafish appear to be maternally provided in medaka, which precluded their analysis; Fig. 4A). For example, in latio hybrids, onset of expression of the paternal medaka ortholog at $\sim 5 \mathrm{hpf}$ temporally matched the onset of expression of the maternal zebrafish ortholog at $\sim 5 \mathrm{hpf}$, but differed from the later onset of expression at $\sim 8 \mathrm{hpf}$ of the same medaka allele in reripes hybrid and purebred medaka embryos (Fig. 4A, B). Thus, the timing of ZGA appears to be largely influenced by factors in the egg and is not intrinsic to the paternal chromatin per se. We therefore conclude that despite having identical genome content, reripes and latio embryos activate their genomes at different times, providing direct evidence for the dominance of cytoplasmic control of the timing of ZGA during embryogenesis. 

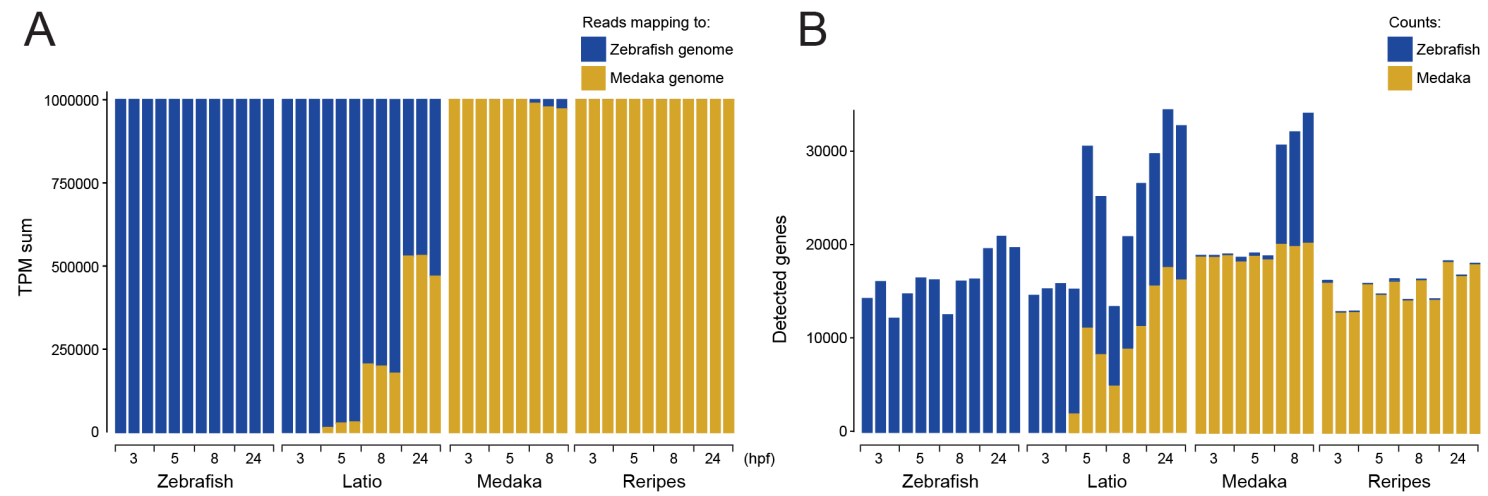

C

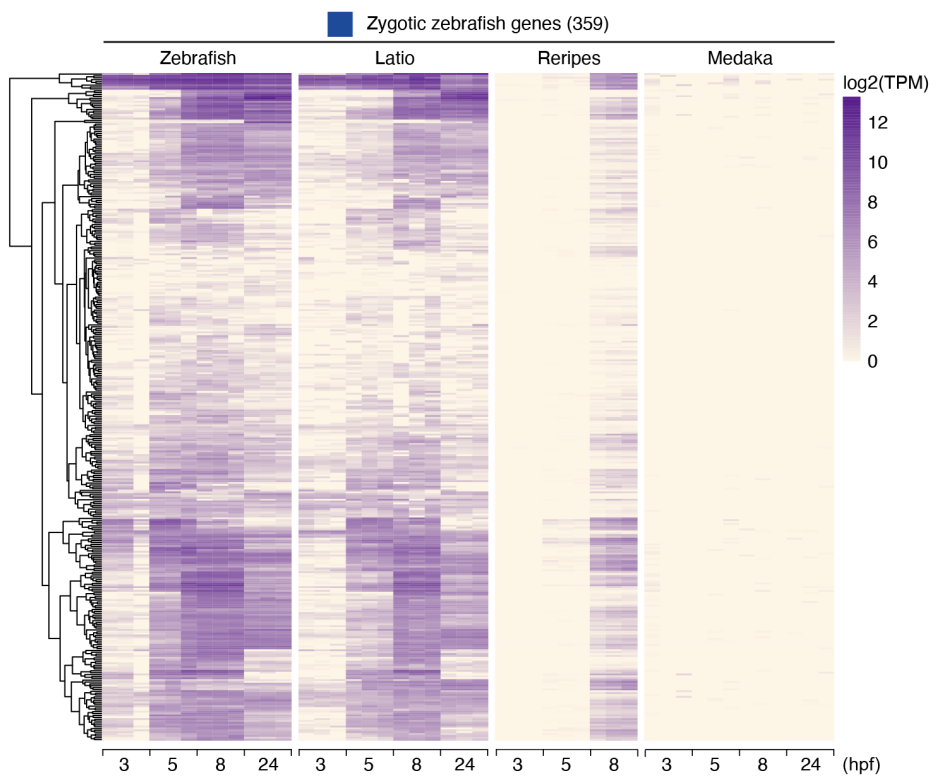

$\mathrm{D}$



Figure 3. The foreign paternal genome is expressed and activated according to the respective egg genome timing in both hybrids.

(A) Total TPM (transcripts per million) mapped to the zebrafish (blue) and medaka (orange) genomes in all samples. The first medaka-derived transcripts are detected at $5 \mathrm{hpf}$ in the 
latio hybrid, whereas zebrafish transcripts are not detected until $8 \mathrm{hpf}$ in reripes.

(B) Number of protein-coding genes detected (read counts $>3$ ) in each sample with genome of origin indicated (zebrafish in dark blue, medaka in dark orange). Upon expression of the foreign paternal genome, the number of detected genes nearly doubles in latio and reripes as a result of both the medaka and zebrafish genomes being expressed.

(C) Heat map of gene expression dynamics in zebrafish, latio, reripes, and medaka embryos for the subset of 359 protein-coding genes that are zygotically expressed in purebred zebrafish. Expression values are plotted as log2(TPM). (D) Heat map of gene expression dynamics in zebrafish, latio, reripes and medaka embryos for the subset of 192 protein-coding genes that are zygotically expressed in purebred medaka. Expression values are plotted as log2(TPM).

A

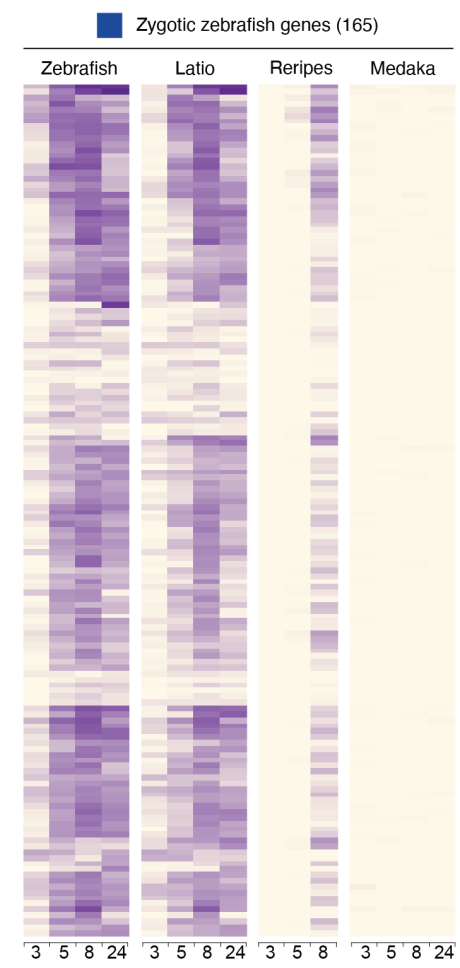

B

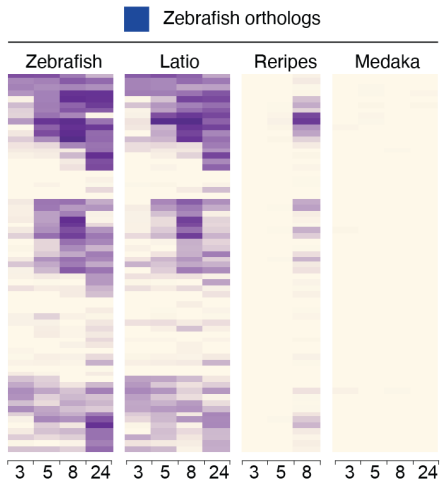

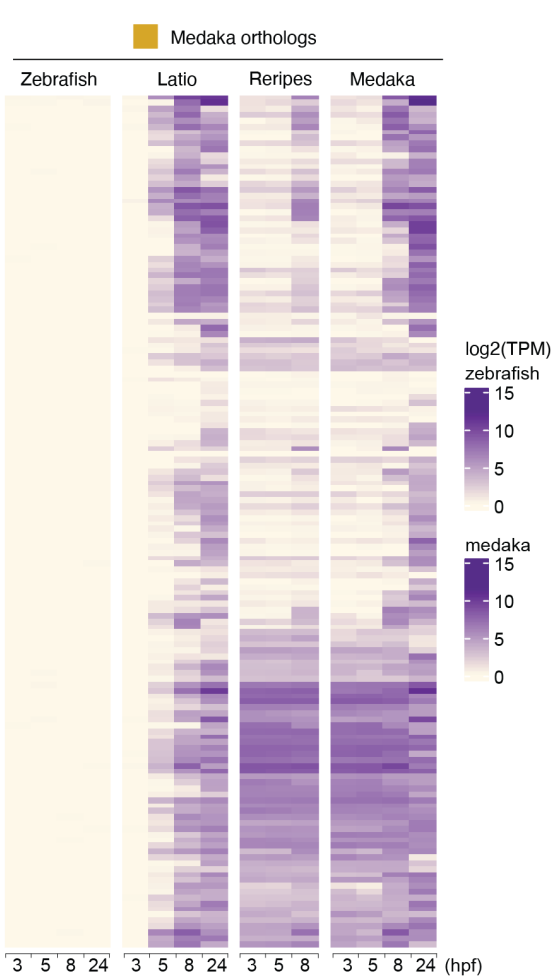

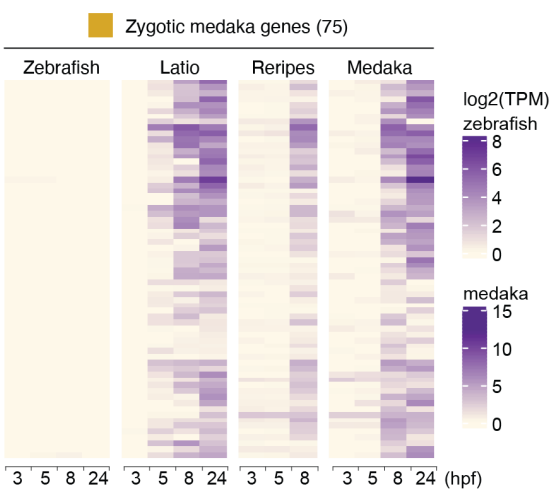

Figure 4. One-to-one orthologs of zygotic genes in zebrafish and medaka follow the expression dynamics of the maternal ortholog.

(A) Heat map of gene expression dynamics of the 165 one-to-one orthologs of zebrafish zygotic genes. Log2(TPM) expression values are shown for zebrafish genes (left) and the 
orthologous medaka genes (right).

(B) Heat map of gene expression dynamics of the 75 one-to-one orthologs of medaka zygotic genes. Log2(TPM) expression values are shown for zebrafish genes (left) and the orthologous medaka genes (right).

\section{Discussion}

Complementary latio and reripes hybrids constitute a distinctive system for studying early development with the advantage of clear differentiation between maternal and paternal components in the embryo. The generation and initial characterization of these hybrids presented here provide insights into both the processes of egg activation and fertilization as well as the control of ZGA timing. We envision this hybrid system as a tool with widespread applications, from studying inheritance of maternal piRNAs or epigenetic marks (Guo et al., 2021), to further probing the intricacies of transcriptional activation during embryogenesis.

Fertilization of zebrafish Bouncer-expressing medaka eggs by zebrafish sperm extends and strengthens our previous finding in the reverse orientation (Herberg et al., 2018). Together, these studies show that compatibility between zebrafish and medaka gametes can be achieved by expressing the sperm-compatible Bouncer protein on the heterospecific egg. Moreover, our findings demonstrate the widespread essentiality of Bouncer as a fertilization factor in fish. However, although Bouncer is required in both species to mediate sperm binding to the egg, zebrafish and medaka have diverged in their modes of egg activation. Given that binding of sperm to the egg via Bouncer is not sufficient to trigger egg activation in medaka, we speculate that PLCZ1, a sperm-provided factor known for its role in egg activation in mammals (Swann \& Lai, 2016; Hachem et al., 2017), may be required in medaka. Consistent with this idea, PLCZ1 is expressed in medaka testis (Ito et al., 2008) but transcripts of the closest zebrafish homolog based on protein sequence (Plcd4a) are absent from zebrafish testis in published RNA-seq data (Herberg et al., 2018; Noda et al., 2021).

Apart from the different requirements for egg activation in zebrafish vs. medaka, we also observed interesting differences between latio and reripes hybrids. Latio hybrids survive for a longer time than reripes hybrids ( 24 vs. $<16$ hours), highlighting asymmetry in the ability of each species' cytoplasm to cope with a haploid foreign genome. Such a phenomenon has been previously observed for paternal Xenopus tropicalis $\mathrm{x}$ maternal $X$. laevis hybrids which are viable, while the reverse cross fails to reach gastrulation (Bürki, 1984; Gibeaux et al., 2018).

The juxtaposition of two differently timed genomes within the same cytoplasm in both parental orientations allowed us to dissect the roles of paternal and maternal contributions in determining ZGA timing. Our results reveal that the paternal genome does not exhibit an intrinsic species timing when placed into a foreign cytoplasm, but instead adopts the timing imposed by the maternal cytoplasm. This is further evident by the fact that even though latio and reripes hybrids are genetically identical, they undergo ZGA at different times, providing direct evidence for cytoplasmic rather than genomic control of ZGA timing during embryogenesis.

The innate differential ZGA timing in medaka and zebrafish begs the question of what cytoplasmic factors drive this difference. Several models have been proposed to explain what regulates ZGA timing, including lengthening of the cell cycle to allow sufficient time for transcription, titration of maternal repressors (such as histones) as the nuclear/cytoplasmic $(\mathrm{n} / \mathrm{c})$ ratio increases, or an increase in concentration of specific transcriptional activators over time (Lee, Bonneau, \& Giraldez, 2014; Pálfy, Joseph, \& Vastenhouw, 2017). In terms of n/c ratio, latio hybrids contain approximately $75 \%$ of the total DNA content normally present in an unaltered zebrafish cytoplasm, effectively decreasing the $n / c$ ratio. Our observations of normal 
ZGA for the zebrafish genome and the $\sim 5 \mathrm{hpf}$ onset of expression for the medaka genome in latio hybrids argue against a major role for $n / c$ ratio in ZGA onset (Fig. 3C, D and Fig. 4). An even stronger case can be made for the reripes hybrids that, despite containing $\sim 150 \%$ of the normal DNA content in a medaka embryo, do not exhibit a shift toward earlier ZGA onset (Fig. 3C, D and Fig. 4).

However, from the perspective of competition between histones as maternal repressors and transcription factors as maternal activators of transcription (Joseph et al., 2017), one could speculate that the early-activating zebrafish cytoplasm tips this balance in favor of transcription sooner than medaka, allowing earlier ZGA. In zebrafish, it has been shown that the transcription factors Pou5f3, Sox $19 \mathrm{~b}$, and Nanog are required for activating expression of the earliest zygotic genes (Lee et al., 2013), and that the concentration of free histones in the nucleus decreases with ZGA onset (Joseph et al., 2017), thus allowing transcription factor binding. Transcriptional competency of the earliest transcribed genes has been shown to be mediated by H3K27Ac writing and reading by P300 and Brd4, respectively (Chan et al., 2019). Analogous studies have not yet been performed in medaka, raising the intriguing possibility that the histone/transcription factor balance is shifted to favor later onset of ZGA due to currently unexplored mechanisms. However, it has been shown that RNA polymerase II is phosphorylated in most cells of the medaka embryo at approximately $4 \mathrm{hpf}$ (128 cells) and that cell divisions lose their synchrony prior to the mid-blastula transition (in contrast to zebrafish) (Kraeussling, Wagner, \& Schartl, 2011), suggesting that medaka development may be governed by factors not shared by zebrafish. Further studies characterizing the cytoplasmic mechanisms that underlie the differential timing between genetically identical latio and reripes hybrid embryos will shed light on the basis for the different developmental timing in zebrafish and medaka, and more generally on species-specific differences in core embryonic programs. 


\section{Materials and Methods}

\section{Zebrafish and medaka husbandry}

Wild-type medaka fish (Oryzias latipes, CAB strain) were raised according to standard protocols $\left(28^{\circ} \mathrm{C}\right.$ water temperature; $14 / 10$ hour light/dark cycle) and served as wild-type medaka. Oryzias curvinotus were obtained from the National Institute for Basic Biology (NIBB) (Okazaki, Japan) and raised under the same conditions as O. latipes. Transgenic zebrafish Bouncer-expressing medaka lines were generated as part of this study and are described in detail below. Zebrafish (Danio rerio) were raised according to standard protocols $\left(28^{\circ} \mathrm{C}\right.$ water temperature; $14 / 10$ hour light/dark cycle). TLAB fish, generated by crossing zebrafish $A B$ and the natural variant TL (Tupfel Longfin) stocks, served as wild-type zebrafish for all experiments. Bouncer mutant zebrafish and medaka Bouncer-expressing transgenic zebrafish lines had been published previously (Herberg et al., 2018).

All animal experiments were conducted according to Austrian and European guidelines for animal research and approved by the Amt der Wiener Landesregierung, Magistratsabteilung 58 - Wasserrecht (animal protocols GZ 342445/2016/12 and MA 58-221180-2021-16 for work with zebrafish; animal protocol GZ: 198603/2018/14 for work with medaka).

\section{Generation of transgenic medaka fish}

The ubiquitin promoter and zebrafish bouncer N-terminally tagged with sfGFP (Herberg et al., 2018) were introduced into the pBluescript II SK(-) vector containing I-Scel sites (gift from Katharina Lust) via Gibson cloning. An injection mix containing ubi::sfGFP-zebrafish Bouncer plasmid (10 ng/ $\mu \mathrm{L})$, I-Scel meganuclease (1:10), CutSmart buffer $(0.5 \mathrm{X})$, Yamamoto's ringer's solution (1X: $1.00 \mathrm{~g} \mathrm{NaCl}, 0.03 \mathrm{~g} \mathrm{KCl}, 0.04 \mathrm{~g} \mathrm{CaCl}_{2} \cdot 2 \mathrm{H}_{2} \mathrm{O}, 0.10 \mathrm{~g} \mathrm{MgCl}_{2} \cdot 6 \mathrm{H}_{2} \mathrm{O}, 0.20 \mathrm{~g} \mathrm{NaHCO}_{3}$ in $1000 \mathrm{~mL}, \mathrm{pH} 7.3)(0.5 \mathrm{X})$, and phenol red (1:5) was prepared and incubated at room temperature for 1 hour before being placed on ice. Wild-type one-cell medaka embryos were collected from natural medaka crosses and microinjected with $1 \mathrm{~nL}$ of injection mix on ice in a medaka injection mold made from $2 \%$ agarose in $1 \mathrm{X}$ Yamamoto's ringer solution. Embryos were kept at $28^{\circ} \mathrm{C}$ and screened for fluorescence 3 or more days post-injection. Fluorescent embryos were reared to adulthood and crossed to wild-type fish to identify founders and generate F1 transgenic fish.

\section{In vitro fertilization with medaka and zebrafish}

Wild-type TLAB zebrafish males were set up the night before experimentation with wild-type zebrafish females in a small, plastic breeding tank with a divider separating the two fish. Medaka crosses were set up the night before experimentation inside their tanks in the fish water system with a vertical divider separating one male from two to three females or two males from four to five females. For generation of unfertilized, unactivated medaka eggs, infertile hybrid $O$. curvinotus $\times O$. latipes males were used to mate with wild-type $C A B$ females or transgenic CAB females expressing zebrafish Bouncer (tg[ubi::sfGFP-zebrafish Bouncer]). On the day of experimentation, sperm was collected from the zebrafish males after anesthetization in $0.1 \%(\mathrm{w} / \mathrm{v})$ tricaine $\left(25 \mathrm{X}\right.$ stock solution in $\mathrm{dH}_{2} \mathrm{O}$, buffered to $\mathrm{pH} 7-7.5$ with 1 $\mathrm{M}$ Tris $\mathrm{pH}$ 9.0) in fish system water. A capillary fitted with small plastic tubing and a pipette filter tip on the other end was used to mouth-pipette sperm from the urogenital opening of each male positioned belly-up in a slit in a sponge wetted with fish water. Sperm was transferred directly to a 1.5-mL tube containing Hank's balanced salt solution (see below) on ice. In general, based on the number of clutches to be fertilized, one male was used per 100 $\mu \mathrm{L}$ of Hank's saline. Because sperm is used in great excess during IVF, any concentration above 50,000 sperm/ul was used.

Freshly spawned medaka eggs were collected directly from the bodies of females using a net with fine mesh and by gently pulling the eggs from the fish in the net using the thumb and forefinger on the outside of the net, taking care not to crush any eggs during removal. Collected eggs were placed into petri dishes containing 1X Yamamoto's ringer's solution. After collection, eggs were visually inspected under a dissection microscope to remove any crushed or already activated eggs. As much ringer's solution as possible was removed from each dish 
such that the eggs remained submerged when the dish was tilted on its lid. $45 \mu \mathrm{L}$ of sperm suspension was pipetted directly onto the eggs. After 2 minutes, $2-3 \mu \mathrm{L}$ of $0.1 \%(\mathrm{w} / \mathrm{v})$ calcimycin in DMSO was pipetted carefully onto the eggs. After 10 minutes, the dishes were filled with E3 medium ( $5 \mathrm{mM} \mathrm{NaCl}, 0.17 \mathrm{mM} \mathrm{KCl}, 0.33 \mathrm{mM} \mathrm{CaCl}_{2}, 0.33 \mathrm{mM} \mathrm{MgSO}_{4}, 0.00001 \%$ methylene blue) or 1X Yamamoto's ringer's solution before being placed into an incubator at $28^{\circ} \mathrm{C}$.

Confocal imaging of zebrafish and medaka sperm in the medaka micropyle

Zebrafish sperm and medaka eggs were collected as described above for IVF. To label sperm, MitoTracker Deep Red (1:400) was added to the Hank's solution before sperm collection. Unactivated medaka eggs (one per fertilization movie) were placed into a medaka injection mold made with $2 \%$ agarose in $1 \mathrm{X}$ Yamamoto's ringer solution in a standard petri dish and positioned with a metal probe under a dissection microscope so that the micropyle was visible. The mold containing the positioned egg was then placed onto an LSM800 Examiner Z1 (Zeiss) upright confocal microscope and the egg was imaged with a 20x/1.0 plan-apochromat water objective. Imaging was started and 6 million sperm were pipetted as close as possible to the egg under the objective. For experiments with calcimycin, $2-3 \mu \mathrm{L}$ of $0.1 \%$ calcimycin in DMSO was pipetted as near to the egg as possible 1.5-2 minutes (such that sperm had reached the end of the micropyle) post sperm addition. During imaging, the micropyle was in focus at all times until sperm fusion and sperm were followed by manually adjusting focus as they moved through the micropyle and if they fused with the egg.

\section{Bright field imaging}

Live embryos were imaged in their chorions in $1.5 \%$ methylcellulose on a glass slide using a dissection microscope (Stemi 508, Zeiss) at 4X magnification using FlyCapture2 (Point Grey Research) and a BlackFly USB color camera (BFLY-U3-23S6C-C).

\section{RNA-seq library preparation and analysis}

Embryo samples for latio hybrids were generated as described in Herberg et al., 2018. Wildtype medaka CAB and zebrafish TLAB embryos were collected from natural crosses after being separated the night before sample collection. Reripes samples were generated as described above. All samples were collected in triplicates at each time point $(3,5,8$, and 24 hpf (except $24 \mathrm{hpf}$ for reripes hybrids). To isolate RNA, samples of 10 embryos per time point were homogenized in TRIzol with an electric pestle and flash-frozen in liquid nitrogen. RNA isolation was performed using standard protocols (phenol/chloroform extraction followed by isopropanol precipitation). RNA concentration was measured using a Fragment Analyzer System (Agilent). rRNA depletion was performed using either the RiboCop rRNA Depletion Kit (Lexogen) using $500 \mathrm{ng}$ of RNA per sample (zebrafish, latio and medaka samples) or the Ribo-zero gold kit (reripes samples). Libraries were prepared using NEBNext Ultra Directional RNA Library Prep Kit for Illumina (NEB) (zebrafish, latio and medaka samples) or the Illumina TruSeq Stranded Total RNA Library Prep kit (reripes samples) and checked using a Fragment Analyzer System (Agilent) before multiplexing. Sequencing was performed on Illumina HiSeqV4 SR100 and NovaSeq 150 bp paired-end sequencing platform.

\section{RNA-seq analysis}

RNA-seq raw reads were adapter trimmed using bbmap v38.26 and mapped to a D.rerio and $O$. latipes hybrid reference genome composed of the Ensembl build 96 genomes GRCz11 and OIASM223467 using Hisat2 v2.1.0. Reads in genes were counted using htseq v0.11.0 ($\mathrm{m}$ intersection-nonempty). TPM estimates were derived from gene-level counts by normalizing for gene length, sequencing depth, and scaling to the sum of 1 million. Only uniquely mapping reads were used for analysis (genes for which reads mapped to the foreign genome in the purebred fish species with an expression level TPM $>5$ were filtered out). Orthologous genes were obtained from Ensembl Compara with homology type ortholog_one2one. D. rerio zygotic gene candidates were defined based on a SlamSeq embryonic time course (Bhat et al., in preparation). Candidate $O$. latipes zygotic genes were defined as genes that are not detected 
at stage $6($ TPM $<0.001)$ but are detected at stage $11(\mathrm{TPM}>2)$ based on GSE136018 (Li et al., 2020).

\section{Conflict of Interest Statement}

The authors declare that the research was conducted in the absence of any commercial or financial relationships that could be construed as a potential conflict of interest.

\section{Author Contributions}

KRG and AP conceived the study; KRG designed, performed, and analyzed experiments; LECQ and MN performed computational analyses of the RNA-seq data; YG and BRC contributed to RNA-seq data generation; AP coordinated and supervised the project; KRG and $\mathrm{AP}$ wrote the manuscript with input from all authors.

\section{Funding}

This research was funded by the Research Institute of Molecular Pathology (IMP), Boehringer Ingelheim, the Austrian Academy of Sciences, FFG (Headquarter grant FFG-852936), the FWF START program (Y 1031-B28) to AP, the HFSP Career Development Award (CDA00066/2015) and the HFSP Young Investigator Award to AP, EMBO-YIP funds to AP, a DOC fellowship from the Austrian Academy of Sciences (OeAW) to KRG, a Boehringer Ingelheim Fonds (BIF) PhD fellowship to LECQ, and Howard Hughes Medical Institute to BRC. The funders had no role in study design, data collection and analysis, decision to publish, or preparation of the manuscript. For the purpose of Open Access, the author has applied a CC BY public copyright license to any Author Accepted Manuscript (AAM) version arising from this submission.

\section{Acknowledgments}

We thank Katharina Lust (IMP Vienna, Austria) for providing a plasmid and valuable advice for working with medaka; Kiyoshi Naruse (Okazaki, Japan) for helpful advice, and the National Institute for Basic Biology (NIBB) (Okazaki, Japan) for providing O. curvinotus; the team of the BioOptics facility at the Vienna Biocenter, in particular Pawel Pasierbek for support with microscopy; the Next Generation Sequencing facility at the Vienna BioCenter Core Facilities (VBCF) for sequencing; the animal facility personnel from the IMP for taking excellent care of zebrafish and medaka; and the entire Pauli lab, particularly Victoria Deneke and Jessica Stock, for fruitful discussions.

\section{Ethics Statement}

All animal experiments were conducted according to Austrian and European guidelines for animal research and approved by the Amt der Wiener Landesregierung, Magistratsabteilung 58 - Wasserrecht (animal protocols GZ 342445/2016/12 and MA 58-221180-2021-16 for work with zebrafish; animal protocol GZ: 198603/2018/14 for work with medaka).

\section{Data availability statement}

The original contributions presented in the study are included in the article/supplementary material; RNA-Seq data from this study were deposited at Gene Expression Omnibus under accession number GSExxxx; all inquiries can be directed to the corresponding author. 


\section{References}

Herberg, S., Gert, K.R., Schleiffer, A., \& Pauli, A. (2018). The Ly6/uPAR protein Bouncer is necessary and sufficient for species-specific fertilization. Science, 361(6406): 1029-1033.

Harvey, S.A., Sealy, I., Kettleborough, R., Fenyes, F., White, R., Stemple, D., \& Smith, J.C. (2013). Identification of the zebrafish maternal and paternal transcriptomes. Development, 140, 2703-2710. doi:10.1242/dev.095091.

Petkov, P.M., Ding, Y., Cassell, M.A., Zhang, W., Wagner, G., Sargent, E.E., Asquith, S., Crew, V., Johnson, K.A., Robinson, P., Scott, V.E., \& Wiles, M.V. (2004). An efficient SNP system for mouse genome scanning and elucidating strain relationships. Genome Res., Sep;14(9):1806-11. doi: 10.1101/gr.2825804.

Lilue, J., Doran, A. G., Fiddes, I. T., Abrudan, M., Armstrong, J., Bennett, R., Chow, W., Collins, J., Collins, S., Czechanski, A., Danecek, P., Diekhans, M., Dolle, D. D., Dunn, M., Durbin, R., Earl, D., Ferguson-Smith, A., Flicek, P., Flint, J., Frankish, A., ... Keane, T. M. (2018). Sixteen diverse laboratory mouse reference genomes define strain-specific haplotypes and novel functional loci. Nature Genetics, 50(11), 1574-1583. https://doi.org/10.1038/s41588-018-0223-8.

Furutani-Seiki, M., \& Wittbrodt, J. (2004). Medaka and zebrafish, an evolutionary twin study. Mech. Dev., 121(7-8): 629-637.

Wittbrodt, J., Shima, A. \& Schartl, M. (2002). Medaka - a model organism from the far east. Nat Rev Genet, 3, 53-64. https://doi.org/10.1038/nrg704.

Kasahara, M., Naruse, K., Sasaki, S., Nakatani, Y., Qu, W., Ahsan, B., Yamada, T., Nagayasu, Y., Doi, K., Kasai, Y., Jindo, T., Kobayashi, D., Shimada, A., Toyoda, A., Kuroki, Y., Fujiyama, A., Sasaki, T., Shimizu, A., Asakawa, S., Shimizu, N., ... Kohara, Y. (2007). The medaka draft genome and insights into vertebrate genome evolution. Nature, 447(7145), 714-719. https://doi.org/10.1038/nature05846.

Howe, K., Clark, M. D., Torroja, C. F., Torrance, J., Berthelot, C., Muffato, M., Collins, J. E., Humphray, S., McLaren, K., Matthews, L., McLaren, S., Sealy, I., Caccamo, M., Churcher, C., Scott, C., Barrett, J. C., Koch, R., Rauch, G. J., White, S., Chow, W., ... Stemple, D. L. (2013). The zebrafish reference genome sequence and its relationship to the human genome. Nature, 496(7446), 498-503. https://doi.org/10.1038/nature12111.

Westerfield, M. (2007). THE ZEBRAFISH BOOK, 5th Edition; A guide for the laboratory use of zebrafish (Danio rerio), Eugene, University of Oregon Press. Paperback.

Kamei, Y., Itou, J., Oda, S., Masui, M., Kim, J.-H., Ishikawa, T., Yuba, S., Kinoshita, M., Mitani, $\mathrm{H}$. and Todo, T. (2007). Development of a convenient in vitro fertilization method using interspecific hybrids between Oryzias latipes and Oryzias curvinotus. Dev Growth Differ., 49: 721-730. https://doi.org/10.1111/j.1440-169X.2007.00966.x.

Wolenski, J. S., \& Hart, N. H. (1987). Scanning electron microscope studies of sperm incorporation into the zebrafish (Brachydanio) egg. J. Exp. Zool., 243(2), 259-273. https://doi.org/10.1002/jez.1402430211.

Iwamatsu, T., Ishijima, S. \& Nakashima, S. (1993). Movement of spermatozoa and changes in micropyles during fertilization in medaka eggs. J. Exp. Zool., 266: 57-64. https://doi.org/10.1002/jez.1402660109. 
Iwamatsu, T., Onitake, K., Matsuyama, K., Satoh, M., \& Yukawa, S. (1997). Effect of Micropylar Morphology and Size on Rapid Sperm Entry into the Eggs of the Medaka. Zool. Sci, 14(4), 623-628. https://doi.org/10.2108/zsj.14.623.

Cosson J. (2004). The ionic and osmotic factors controlling motility of fish spermatozoa. Aquac Int, 12(1):69-85.

Iwamatsu, T., Onitake, K., Yoshimoto, Y. and Hiramoto, Y. (1991), Time Sequence of Early Events in Fertilization in the Medaka Egg. Dev. Growth Differ., 33: 479-490. https://doi.org/10.1111/j.1440-169X.1991.00479.x

Ridgway, E. B., Gilkey, J. C., \& Jaffe, L. F. (1977). Free calcium increases explosively in activating medaka eggs. Proceedings of the National Academy of Sciences of the United States of America, 74(2), 623-627. https://doi.org/10.1073/pnas.74.2.623.

Gilkey, J.C., Jaffe, L.F., Ridgway, E.B., \& Reynolds, G.T. (1978). A free calcium wave traverses the activating egg of the medaka, Oryzias latipes. J Cell Biol, 76(2): 448-466.

Luckasen, J. R., White, J. G., \& Kersey, J. H. (1974). Mitogenic properties of a calcium ionophore, A23187. Proceedings of the National Academy of Sciences of the United States of America, 71(12), 5088-5090. https://doi.org/10.1073/pnas.71.12.5088.

Iwamatsu, T. (2004). Stages of normal development in the medaka Oryzias latipes. Mech. Dev., 121(7-8): 605-618.

Vastenhouw, N. L., Cao, W. X., \& Lipshitz, H. D. (2019). The maternal-to-zygotic transition revisited. Development, 146(11), dev161471. https://doi.org/10.1242/dev.161471.

Aizawa, K., Shimada, A., Naruse, K., Mitani, H., \& Shima, A. (2003). The medaka midblastula transition as revealed by the expression of the paternal genome. Gene Expr. Patterns, 3(1): 43-47. https://doi.org/10.1016/S1567-133X(02)00075-3.

Nakamura, R., Motai, Y., Kumagai, M., Wike, C. L., Nishiyama, H., Nakatani, Y., Durand, N. C., Kondo, K., Kondo, T., Tsukahara, T., Shimada, A., Cairns, B. R., Aiden, E. L., Morishita, S., \& Takeda, H. (2021). CTCF looping is established during gastrulation in medaka embryos. Genome Res 31(6), 968-980. https://doi.org/10.1101/gr.269951.120.

Pauli, A., Valen, E., Lin, MF., Garber, M., Vastenhouw, NL., Levin, JZ., Fan, L., Sandelin, A., Rinn, JL., Regev, A., Schier, AF. (2012). Systematic identification of long noncoding RNAs expressed during zebrafish embryogenesis. Genome Res, 22(3):577-91. doi:10.1101/gr.133009.111.

Chew, GL., Pauli, A., Rinn, JL., Regev, A., Schier, AF., Valen, E. (2013). Ribosome profiling reveals resemblance between long non-coding RNAs and 5' leaders of coding RNAs. Development 140(13):2828-34. doi:10.1242/dev.098343.

Li, Y., Liu, Y., Yang, H., Zhang, T., Naruse, K., \& Tu, Q. (2020). Dynamic transcriptional and chromatin accessibility landscape of medaka embryogenesis. Genome Res, 30(6), 924-937. https://doi.org/10.1101/gr.258871.119

Guo, Y., Gert, K.R., Lebedeva, S., Potok, M.E., Wike, C.L., Grow, E.J., Ketting, R.F., Pauli, A., \& Cairns, B.R. (2021). Maternally-inherited anti-sense piRNAs antagonize transposon expression in teleost embryos. bioRxiv. 
Swann, K., \& Lai, F. A. (2016). Egg Activation at Fertilization by a Soluble Sperm Protein. Physiological reviews, 96(1), 127-149. https://doi.org/10.1152/physrev.00012.2015.

Hachem, A., Godwin, J., Ruas, M., Lee, H. C., Ferrer Buitrago, M., Ardestani, G., Bassett, A., Fox, S., Navarrete, F., de Sutter, P., Heindryckx, B., Fissore, R., \& Parrington, J. (2017). PLC\} is the physiological trigger of the Ca2+ oscillations that induce embryogenesis in mammals but conception can occur in its absence. Development, 144(16), 2914-2924. https://doi.org/10.1242/dev.150227.

Ito, M., Shikano, T., Oda, S., Horiguchi, T., Tanimoto, S., Awaji, T., Mitani, H., \& Miyazaki, S. (2008). Difference in Ca2+ oscillation-inducing activity and nuclear translocation ability of PLCZ1, an egg-activating sperm factor candidate, between mouse, rat, human, and medaka fish. Biol. Reprod, 78(6), 1081-1090. https://doi.org/10.1095/biolreprod.108.067801.

Noda, T., Blaha, A., Fujihara, Y., Gert, K.R., Emori, C., Deneke, V.E., Oura, S., Berent, S., Kodani, M., Panser, K., Cabrera Quio, L.E., Pauli, A., \& Ikawa, M. (2021). Sperm membrane proteins DCST1 and DCST2 are required for the sperm-egg fusion process in mice and fish. bioRxiv. doi: https://doi.org/10.1101/2021.04.18.440256.

Bürki, E. (1985). The expression of creatine kinase isozymes in Xenopus tropicalis, Xenopus laevis laevis, and their viable hybrid. Biochem. Genet., 23, 73-88.

Gibeaux, R., Acker, R., Kitaoka, M., Georgiou, G., van Kruijsbergen, I., Ford, B., Marcotte, E. M., Nomura, D. K., Kwon, T., Veenstra, G., \& Heald, R. (2018). Paternal chromosome loss and metabolic crisis contribute to hybrid inviability in Xenopus. Nature, 553(7688), 337-341. https://doi.org/10.1038/nature25188.

Lee, M. T., Bonneau, A. R., \& Giraldez, A. J. (2014). Zygotic genome activation during the maternal-to-zygotic transition. Annual review of cell and developmental biology, 30, 581-613. https://doi.org/10.1146/annurev-cellbio-100913-013027.

Pálfy, M., Joseph, S.R., \& Vastenhouw, N.L. (2017). The timing of zygotic genome activation. Curr. Opin. Genet. Dev., 43, 53-60. https://doi.org/10.1016/j.gde.2016.12.001.

Joseph, S. R., Pálfy, M., Hilbert, L., Kumar, M., Karschau, J., Zaburdaev, V., Shevchenko, A., \& Vastenhouw, N. L. (2017). Competition between histone and transcription factor binding regulates the onset of transcription in zebrafish embryos. eLife, 6, e23326. https://doi.org/10.7554/eLife.23326

Lee, M. T., Bonneau, A. R., Takacs, C. M., Bazzini, A. A., DiVito, K. R., Fleming, E. S., \& Giraldez, A. J. (2013). Nanog, Pou5f1 and SoxB1 activate zygotic gene expression during the maternal-to-zygotic transition. Nature, 503(7476), 360-364.

https://doi.org/10.1038/nature12632

Chan, S. H., Tang, Y., Miao, L., Darwich-Codore, H., Vejnar, C. E., Beaudoin, J. D., Musaev, D., Fernandez, J. P., Benitez, M., Bazzini, A. A., Moreno-Mateos, M. A., \& Giraldez, A. J. (2019). Brd4 and P300 Confer Transcriptional Competency during Zygotic Genome Activation. Dev. Cell, 49(6), 867-881.e8. https://doi.org/10.1016/j.devcel.2019.05.037

Kraeussling, M., Wagner, T. U., \& Schartl, M. (2011). Highly asynchronous and asymmetric cleavage divisions accompany early transcriptional activity in pre-blastula medaka embryos. PloS One, 6(7), e21741. https://doi.org/10.1371/journal.pone.0021741. 